\title{
Therapeutic effects of sesamolin on leukemia induced by WEHI-3B in model mice
}

\author{
Senthil Nagarajan ${ }^{1,2}$ and Jae Kwon Lee ${ }^{1 *}$ (D)
}

\begin{abstract}
Sesamolin is one of the lignans derived from sesame oil. It has demonstrated significant antioxidant, anti-aging, and anti-mutagenic properties. It also reportedly augments natural killer (NK) cell lysis activity. We previously reported that sesamolin also exerts anticancer effects in vitro and induces enhanced NK cell cytolytic activity against tumor cells. Herein, we aimed to determine the mechanism by which sesamolin prevents and retards tumorigenesis in BALB/C mouse models of leukemia induced by murine (BALB/C) myelomonocytic leukemia WEHI-3B cells. Banded neutrophils, myeloblasts, and monocytic leukemic cells were more abundant in the leukemia model than in normal mice. Sesamolin decreased the number of leukemic cells by almost 60\% in the leukemia model mice in vivo; additionally, sesamolin and the positive control drug, vinblastine, similarly hindered neoplastic cell proliferation. Spleen samples were $\sim 4.5$-fold heavier in leukemic mice than those obtained from normal mice, whereas spleen samples obtained from leukemic mice treated with sesamolin had a similar weight to those of normal mice. Moreover, sesamolin induced a twofold increase in the cytotoxic activity of leukemic mouse NK cells against WEHI-3B cells. These results indicated that sesamolin exerts anti-leukemic effects in vivo.
\end{abstract}

Keywords: Cancer, Therapy, Sesamolin, Leukemia, Myeloblast, Sesame

\section{Introduction}

Cancer is generally defined as atypical cell proliferation, and the hallmarks of tumor development comprise insensitivity to anti-growth signals, metastasis, apoptotic evasion, unlimited replicative potential, and sustained angiogenesis $[1,2]$. The most common cause of cancer is chronic inflammation [3], during which the immune system is tasked with releasing protective agents, such as reactive oxygen species and nitric oxide, to counter pathogens [4]. These agents disrupt the DNA repair mechanisms, which can result in DNA mutations [3].

Leukemia is a type of blood cancer that generally arises in the bone marrow and results in an overabundance of immature leukocytes. The main symptoms of leukemia include bruising, bleeding, fever, and high susceptibility to infections [5]. Leukemia comprises both the

\footnotetext{
*Correspondence: chemokine@cbnu.ac.kr

1 Department of Biology Education, College of Education, Chungbuk National University, Cheongju, Chungbuk 361-763, Republic of Korea Full list of author information is available at the end of the article
}

lymphocytic type and the myeloid type, according to the category of overly proliferating blood cells. Myeloid leukemia is characterized by the rapid growth of myeloid cells and immature granulocytes that accumulate in the bone marrow and blood [6]. Herein, we induced a leukemia model by intraperitoneally injecting $\mathrm{BALB} / \mathrm{c}$ mice with WEHI-3B cells.

Sesamolin is a representative lignan in sesame oil [7] that demonstrates verified antioxidative effects [8]. We previously showed that sesamolin exerts anticancer (antileukemic) effects in an in vitro study. Our results indicated that the increased sensitivity of sesamolin-treated myeloma cells to natural killer (NK) cell lysis is caused by heightened NKG2D ligand expression on the surface of myeloma cells, resulting from a more robust ERK signaling pathway [9]. We also disclosed that sesamolin stimulates NK cell cytolytic activity via phosphorylation of the p38, ERK1/2 and JNK pathways [10]. These findings indicated that sesamolin exerts therapeutic effects against tumors. However, such effects have not been 
demonstrated in vivo. Therefore, the present study aimed to determine whether sesamolin exerts anti-leukemic effects in vivo.

\section{Materials and methods Reagents}

Sesamolin was obtained from Nagara Science (Tokyo, Japan) and all reagents with unspecified sources were purchased from Sigma-Aldrich (Seoul, Republic of Korea).

\section{Preparation of naïve NK cells}

Naïve NK cells were isolated from the spleen of C57/ BL6 mice (Dooyeol Biotech Lab, Republic of Korea). The spleen was flushed with phosphate-buffered saline (PBS), and splenocytes were isolated by passing the obtained fluid through a nylon filter with $70 \mu \mathrm{m}$ pores. NK cells were purified by negative selection with immunomagnetic beads (Miltenyi Biotec, Bergisch Gladbach, Germany). The mice were housed under standard conditions $\left(22^{\circ} \mathrm{C}-24^{\circ} \mathrm{C}, 40 \%-60 \%\right.$ humidity $)$ and fed a standard chow diet (Purina, MO, USA). All experimental protocols in vivo were conducted in accordance with the guidelines provided by the Ethics Committee for Animal Experimentation of Chungbuk National University (Permit Number: CBNUA-1174-18-01, CBNUA-1282-19-01).

\section{Murine WEHI-3B leukemia cells}

The murine $(\mathrm{BALB} / \mathrm{c})$ myelomonocytic leukemia cell line, WEHI-3 (Sigma-Aldrich), was cultured at $37{ }^{\circ} \mathrm{C}$ under a $5 \% \mathrm{CO}_{2}$ atmosphere in high-glucose Dulbecco's Modified Eagle (DMEM) medium (Hyclone Laboratories Inc., Logan, UT, USA), containing $10 \%$ fetal bovine serum (FBS) (Hyclone Laboratories Inc.) in addition to $100 \mathrm{U} /$ $\mathrm{mL}$ penicillin and $100 \mu \mathrm{g} / \mathrm{mL}$ of streptomycin (both purchased from Thermo Fisher Scientific Inc., Waltham, MA, USA).

\section{Establishment of the murine leukemia model}

A leukemia model was established as per the procedures described by He and $\mathrm{Na}$ [11]. Normal and leukemic mice were orally administered with sesamolin. The first trial of BALB/c mice proceeded in two parts. Part 1: Eight normal mice were untreated (control). Part 2: Eight mice were divided into two groups, namely 1 and $2(n=8$ each), and were injected intraperitoneally (i.p.) with $1 \times 10^{5}$ and $1 \times 10^{6}$ WEHI-3B cells, respectively. They were photographed 10 days later. The mice were euthanized immediately thereafter under ether anesthesia, and autopsied.

The second trial comprised two parts. Part 1: Normal BALB/c mice were divided into groups, namely 1 and $2(\mathrm{n}=8$ each), which were untreated (control) or administered with sesamolin $(10 \mathrm{mg} / \mathrm{kg})$ in carboxymethyl cellulose $(\mathrm{CMC})$, respectively. Part 2: Thirty mice were divided as follows: group $3(n=8)$ mice were injected i.p. with $1 \times 10^{5}$ or $1 \times 10^{6}$ WEHI-3B cells (disease control); group $4(\mathrm{n}=8)$ mice were injected i.p. with WEHI-3B cells, then administered with vinblastine $(2.5 \mu \mathrm{g} / \mathrm{kg})$ in CMC (positive control); group $5(\mathrm{n}=8)$ mice were injected with WEHI-3B cells, then administered with sesamolin in CMC. Three doses per week of vinblastine and sesamolin were administered per os (p.o.).

The third trial comprised two parts. Part 1: Normal BALB/c mice $(n=16)$ were divided into two groups $(\mathrm{n}=8$ each). Group 1 was the untreated control, and Group 2 mice were administered with sesamolin $(10 \mathrm{mg} /$ $\mathrm{kg}$ ) in CMC. Part 2: Thirty mice were divided into three groups. Group $3(n=8)$ mice were injected i.p. with $1 \times 10^{5}$ WEHI-3B cells (disease control). Group $4(n=8)$ mice were injected i.p. with WEHI-3B cells, then administered with sesamolin on the same day. Group 5 mice were injected i.p. with WEHI-3B cells, then administered with sesamolin from five days thereafter.

None of the mice spontaneously died after sample inoculation. All mice were euthanized 31 days after injection with WEHI-3B cells under ether anesthesia, following which blood, liver, and spleen samples were collected.

\section{Histopathological assessment of bone marrow smears}

The heads of femurs from the euthanized mice were removed and the marrow was flushed. Bone marrow and blood samples were smeared as per previously described methods [12]. Leukocytes in bone marrow and blood smears stained with Diff-Quick (Thermo Fisher) were morphologically identified, and ratios (\%) of myeloblasts or neoplastic cells were determined by counting 200 nucleated cells using a microscope.

Isolated spleen samples were fixed in $4 \%$ formaldehyde, embedded in paraffin, and sectioned at $5 \mu \mathrm{m}$ thickness for histopathological assessment. The paraffin-embedded sections were mounted on slides glass, stained with hematoxylin and eosin (H\&E) as per methods described previously [12], and then histopathological evaluation was conducted by microscopy at $400 \times$ magnification.

\section{Fluorescence-activated cell sorter (FACS) analysis}

Peripheral blood mononuclear cells (PBMCs) were analyzed by FACS using the FACSCalibur (Becton Dickinson and Co., Franklin Lakes, NJ, USA) as per previously described methods [13]. Briefly, blood was collected from each mouse, and then PBMCs were isolated using a Ficoll density gradient and stained with the fluorescence-labeled monoclonal antibodies (mAb), namely anti-CD3-FITC, anti-CD19-FITC, anti-CD11b-PE, 
anti-Mac-3-FITC, and isotype control antibodies (all purchased from Becton Dickinson and Co.).

\section{NK cell cytotoxicity assays}

The cytolytic ability of NK cells was determined using lactate dehydrogenase (LDH) assays (Takara Bio Inc., Kusatsu, Japan). Cancer (target) $\left(6 \times 10^{4}\right)$ and NK (effector) cells $\left(1.8 \times 10^{5}\right)$ mixed in 96-cell plates were incubated under a $5 \% \mathrm{CO}_{2}$ atmosphere at $37^{\circ} \mathrm{C}$. After $4 \mathrm{~h}$, the plates were centrifuged at $250 \times g$ for $10 \mathrm{~min}$, and supernatants $(100 \mu \mathrm{L})$ were transferred to new 96 -well plates and mixed with the LDH assay substrate $(100 \mu \mathrm{L})$. Assay results were measured as absorbance at $490 \mathrm{~nm}$.

Cytotoxicity was calculated as:

$$
\begin{aligned}
\% \text { cytotoxicity }(\%)= & {[(\mathrm{A}-\text { low control }) /(\text { high control }} \\
& - \text { low control })]
\end{aligned}
$$

where $\mathrm{A}=$ [effector-target cell mix] - [effector-cell control]. Spontaneous LDH release was considered as the amount of activity released from the cancer cells in the low control, and maximal LDH release was measured by adding Triton X-100 to the high control. The effectorcell control measured spontaneous LDH release, which was the amount of LDH activity released from NK cells. The low- and effector-cell control groups were treated with dimethyl sulfoxide (DMSO), which was the vehicle for sesamolin.

\section{Statistics}

Results are expressed as mean \pm SD (standard deviation). Data were statistically analyzed using one-way analyses of variance (ANOVA) followed by Dunnett's post-hoc tests. Values with $\mathrm{P}<0.05$ were considered statistically significant' for better specificity.

\section{Results}

Establishment of leukemia animal model to assess anti-leukemic activity of sesamolin

We established a WEHI-3B-induced leukemia model in the first trial, as described in Materials and Methods. It is shown in Fig. 1A that the abdomens of mice with induced leukemia were swollen with ascites. Moreover, cancerous tissues were found between the peritoneum and abdominal muscles in a few mice (data not shown).

The establishment of the leukemia model was verified by the presence of immature and neoplastic leukocytes
(A)

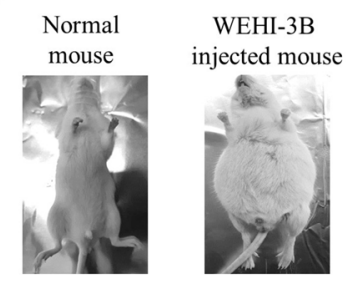

(B)

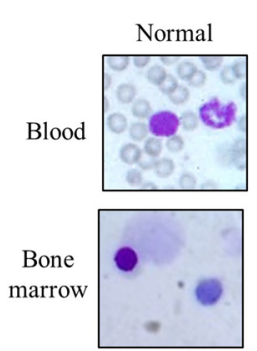

WEHI-3B

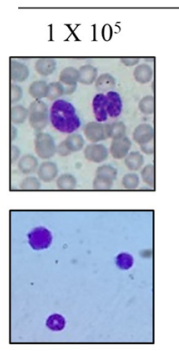

(C)

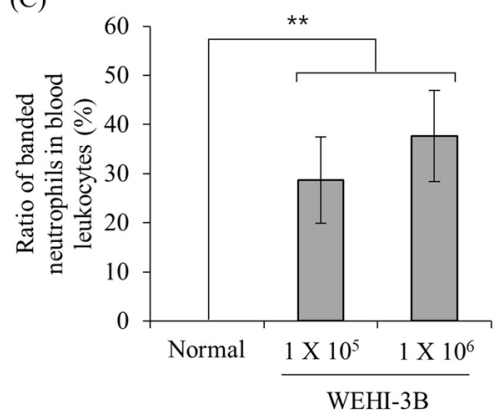

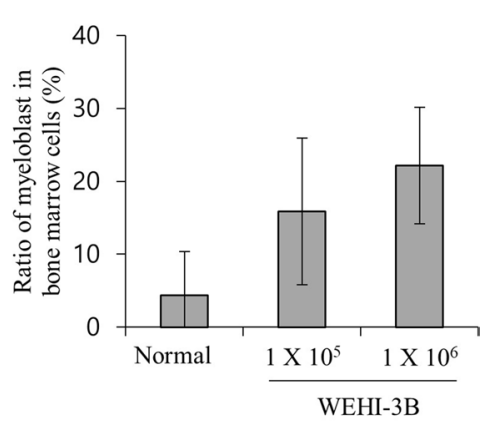

WEHI-3B

(D)

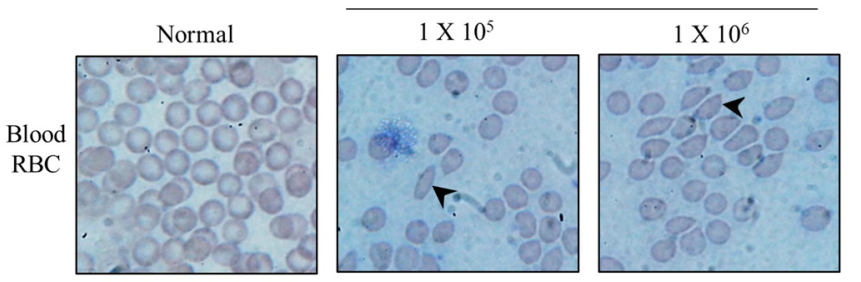

Fig. 1 Generation of leukemic mice with WEHI-3B injection. A Representative images of normal and leukemic BALB/C mouse models. Mice injected with WEHI-3B cells i.p. were photographed in the ventral position 10 days later. B Blood and bone marrow smears from euthanized mice visualized with Wright Giemsa stain. Leukocytes were morphologically classified. Arrows $(\boldsymbol{\rightarrow})$ indicate banded neutrophils and arrows $(\rightarrow)$ indicate myeloblasts. C Ratios (\%) of banded neutrophils and myeloblasts in blood and bone marrow smears. Data from three independent experiments, which included triplicate samples, are shown as mean $\pm S D$. ${ }^{* *} \mathrm{P}<0.01$, leukemic vs. normal mice (one-way ANOVA with Dunnett's post-hoc tests). D Morphological changes in RBCs isolated from leukemic mice. Arrowheads indicate abnormal RBCs isolated from leukemic mice. Data are representative of three independent experiments 
in the blood and bone marrow. Blood and bone marrow smears examined by microscopy 10 days after injecting the mice with WEHI-3B cells revealed several neoplastic cells containing large, irregular, and doughnut-shaped nuclei; conversely, only a few neoplastic cells were evident in normal mice (Fig. 1B). The mice injected with WEHI-3B cells manifested more banded neutrophils and myeloblasts in the blood smear and bone marrow smear, respectively. The ratios (\%) of neoplastic cells in the blood and bone marrow are depicted in Fig. 1C. Banded neutrophils and myeloblasts in the blood smears, and myeloblasts in the bone marrow smears were considered as neoplastic. Although the difference did not demonstrate statistical significance, the number of neoplastic cells increased depending on the number of injected WEHI$3 \mathrm{~B}$ cells.

The biconcave morphology of red blood cells (RBCs) was lost in leukemic mice (Fig. 1D), and the cells appeared longer, uneven, and elliptocytic. It is shown in Fig. 1 that the suitability of the leukemia model induced by WEHI-3B cells was verified for the present study.

\section{Anti-leukemic effect of sesamolin in vivo}

We investigated the anti-leukemic effects of sesamolin in vivo using the WEHI-3B-induced leukemia mouse model established, as enumerated in Table 1 (second trial). The mice were administered with sesamolin and vinblastine (positive control drug) p.o. after leukemia induction was verified.

Figure 2 shows more banded neutrophils, myeloblasts, and immature neutrophils in blood smears from the leukemia model than in the blood smears from normal mice. Leukemic mice treated with sesamolin showed a similar number of malignant hematopoietic cells as leukemic mice treated with vinblastine. Microscopy findings of bone marrow smears also revealed more myeloblasts in leukemic mice than in normal mice, and that sesamolin and vinblastine decreased myeloblast numbers (Fig. 3). The inhibitory effects of sesamolin and vinblastine on neoplastic cell proliferation were similar.

Table 1 Timetable for study of anti-leukemia activity of sesamolin in vivo

\begin{tabular}{|c|c|}
\hline Day & Action \\
\hline 0 & IP injection of $1 \times 10^{5}$ or $1 \times 10^{6} \mathrm{WEHI}-3 \mathrm{~B}$ cells \\
\hline $5-10$ & $\begin{array}{l}\text { Leukemia induction confirmed by direct } \\
\text { peripheral blood and bone marrow smear }\end{array}$ \\
\hline $10-30$ & Sesamolin therapy (3 doses/week) \\
\hline 31 & Sacrifice and autopsy \\
\hline
\end{tabular}

Effects of sesamolin on the weight and histopathology of spleen and liver

Spleen and liver samples from all mice were weighed. Fig. 4A, B show the anticancer effects of sesamolin on organ weight and Fig. 4C shows the histological findings of the spleen. The evidently heavier spleen in the model, compared to that in normal mice, exhibited a reduction in weight, which was induced by sesamolin $(10 \mathrm{mg} /$ $\mathrm{kg}$ ). Spleen samples from leukemic mice contained large, irregular cells, whereas those from leukemic mice treated with sesamolin appeared similar to spleen samples obtained from normal mice (Fig. 4C). Since leukemia induction did not significantly increase liver weight, the anti-leukemic effect of sesamolin could not be confirmed by changes in liver weight. Therefore, histological analysis of liver tissue was not necessary.

\section{Anti-leukemic effects of sesamolin at various time points}

We investigated whether the anti-leukemic effects of sesamolin differed depending on the time of administration. Normal and leukemic mice, classified according to the criteria listed in Table 2, were administered with sesamolin following the schedule depicted in Table 3 (third trial). Group 4 mice were administered with sesamolin on the same day that they were injected with WEHI-3B cells, and group 5 mice were administered with sesamolin after leukemia induction was confirmed. To prevent repeated experimental data for analysis, the vinblastine treatment group was not included in the third trial.

Blood and bone marrow smears (Fig. 5A) showed that sesamolin decreased the number of neoplastic cells (banded neutrophils and myeloblasts) induced by WEHI3B. The ratio (\%) of neoplastic cells expressed as proportions of banded neutrophils and myeloblasts among 200 leukocytes and bone marrow cells, respectively, is depicted in Fig. 5B. More banded neutrophils and myeloblasts were found in leukemic mice than in normal mice. Sesamolin reduced the number of banded neutrophils and myeloblasts in leukemic mice (groups 4 and 5). However, groups 4 and 5 demonstrated similar ratios (\%) of neoplastic cells. These indicated that the anti-leukemic effects of sesamolin are independent of the time of administration.

\section{Impact of sesamolin on the weight of the liver and spleen'} Spleen and liver samples obtained from individual mice from each group on days 5 and 31 were photographed (Fig. 6A) and weighed (Fig. 6B). The spleen samples were longer and wider in leukemic mice compared to the samples obtained from normal mice (Fig. 6A). Moreover, spleen samples were $~ 4.5$-fold heavier in leukemic mice in comparison with the spleen samples from normal 


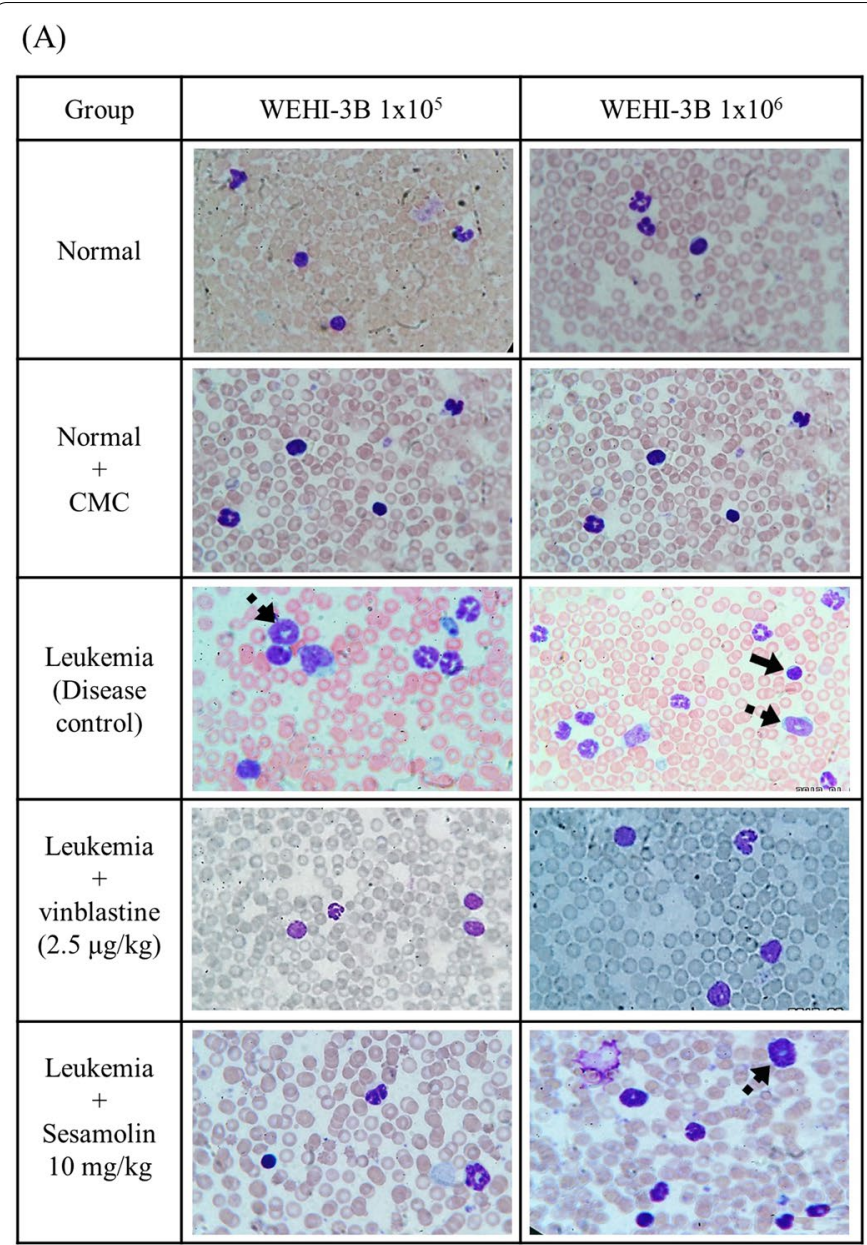

(B)

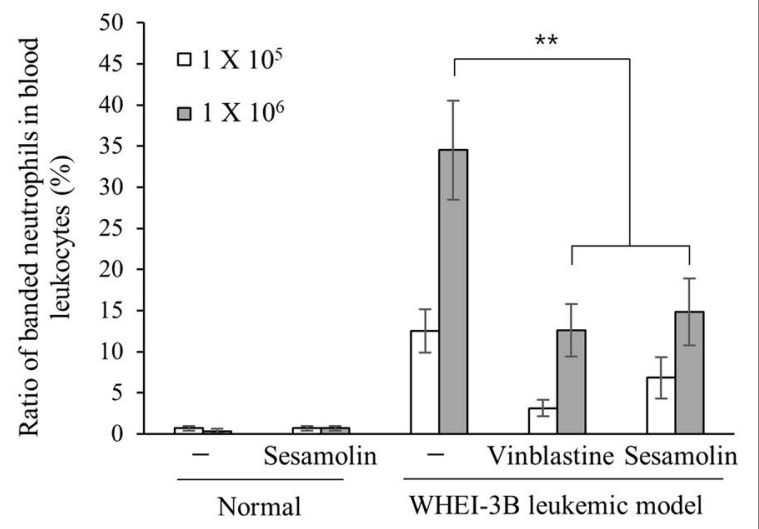

Fig. 2 Blood smears of groups with leukemic mice, which were either administered or not administered with sesamolin. Mice were injected with WEHI-3B, and blood smears prepared 30 days later were visualized with Wright Giemsa staining. A Immature granulocytes and myeloblasts in blood smears. Arrows $(\mathbf{-})$ indicate banded neutrophils and arrows $(\rightarrow)$ indicate myeloblasts. Data are representative of experiments repeated at least three times. B Ratios (\%) of banded neutrophils in total leukocytes. Data from three independent experiments, which included triplicate samples, are shown as mean $\pm S D$. ${ }^{*} P<0.01$ vs. leukemic mice (one-way ANOVA with Dunnett's post-hoc tests)

mice. In Fig. 6B, it is shown that sesamolin exerted similar effects on the spleen when administered on the same day that WEHI-3B cells were injected (group 4) and after tumors were established (group 5). In contrast, leukemia induction did not significantly increase liver weight; thus, the anti-leukemic effects of sesamolin could not be verified in terms of a change in liver weight.

\section{Surface markers of leukocytes in mice}

We used flow cytometry to analyze CD3, CD11b, CD19, and Mac-3, which are surface markers of leukocytes, in blood samples from groups 1, 2, 3, and 5 (Table 2). Leukemia induction significantly decreased the expression of $\mathrm{T}$ (CD3) and B (CD19) cell markers, as shown in Fig. 7. However, sesamolin caused CD3 and CD19 levels to rebound in leukemic mice, and return to levels similar to those of normal mice. Leukemic induction significantly augmented the expression of CD11b and Mac-3, which are markers of monocytes/granulocytes and macrophages, respectively. Furthermore, their respective expression levels were reduced by sesamolin. The results of the markers were similar between normal mice with or without sesamolin administration. These results indicated that sesamolin could recover the ratios of individual types of leukocytes altered by leukemia.

\section{Effects of sesamolin on the cytotoxic activity of naïve NK cells}

The cytotoxic activity of NK cells isolated from groups 1, 2, 3, and 5 (Table 2) was determined using lactate dehydrogenase (LDH) assays of Yac-1 and WEHI3B cells. NK cells derived from normal mice exhibited $10 \%$ and $30 \%$ cytotoxicity against Yac-1 cells and 


\section{(A)}

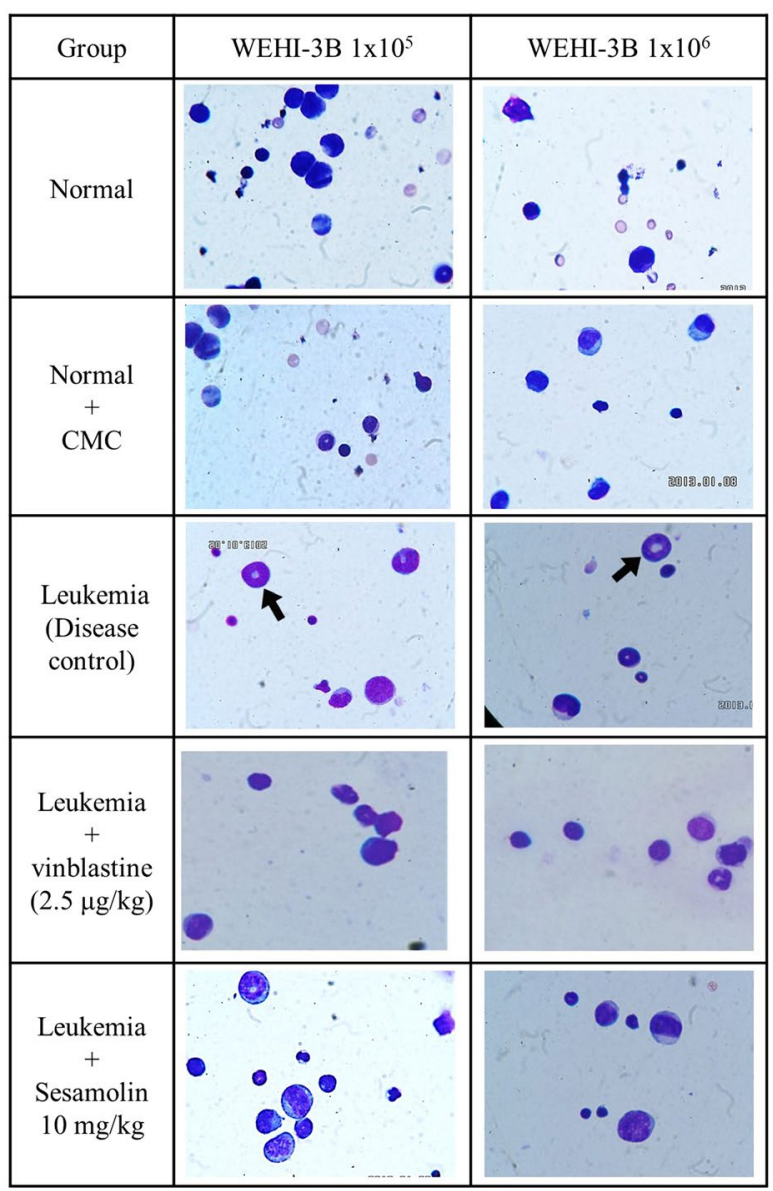

(B)

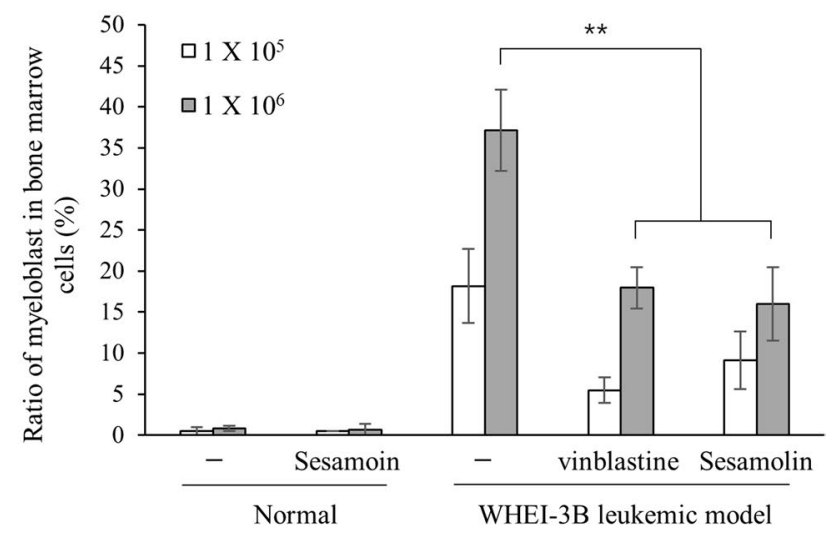

Fig. 3 Bone marrow smears of groups with leukemic mice, which were either administered or not administered with sesamolin. A Myeloblasts in bone marrow smears. B Ratios (\%) of myeloblasts in bone marrow cells. Arrows indicate myeloblasts. Data are representative of experiments repeated at least three times with triplicate samples and are shown as mean $\pm S D$. ${ }^{*} P<0.01$ vs. leukemic mice (one-way ANOVA with Dunnett's post-hoc tests)

Table 2 Experimental groups of mice and treatment

\begin{tabular}{lll}
\hline Group & Mice & Treatment \\
\hline 1 & Normal BALB/c mice & None \\
2 & Normal BALB/c mice & Sesamolin $(10 \mathrm{mg} / \mathrm{kg})$ from day 5 \\
3 & Leukemia model & None \\
4 & Leukemia model & Sesamolin $(10 \mathrm{mg} / \mathrm{kg})$ from day 0 \\
5 & Leukemia model & Sesamolin $(10 \mathrm{mg} / \mathrm{kg})$ from day 5 \\
\hline
\end{tabular}

WEHI-3B cells, respectively, as shown in Fig. 8A. Sesamolin enhanced these activity levels; however, the difference did not attain significance. The activity levels of NK cells derived from leukemic and normal mice were similar to those of Yac-1 cells; however, sesamolin induced a twofold increase in the cytotoxic activity
Table 3 Experimental flow

\begin{tabular}{ll}
\hline Day & Action \\
\hline 0 & $\begin{array}{l}\text { IP injection of } 1 \times 10^{6} \mathrm{WEHI}-3 \mathrm{~B} \text { cells } \\
\text { Commencement of sesamolin (3 doses/week) administration } \\
\text { in group 4 }\end{array}$ \\
$\begin{array}{ll}\text { Confirmation of leukemia induction } \\
10-30\end{array}$ & $\begin{array}{l}\text { Commencement of sesamolin (3 doses/week) administration } \\
\text { in group 5 }\end{array}$ \\
31 & Sacrifice and autopsy \\
\hline
\end{tabular}

of leukemic mouse NK cells against WEHI-3B cells (Fig. 8B).

We investigated the reliability of the results shown in Fig. 8A, B. NK cells from normal mice were incubated with sesamolin $(10 \mu \mathrm{g} / \mathrm{mL})$ for $48 \mathrm{~h}$, following 


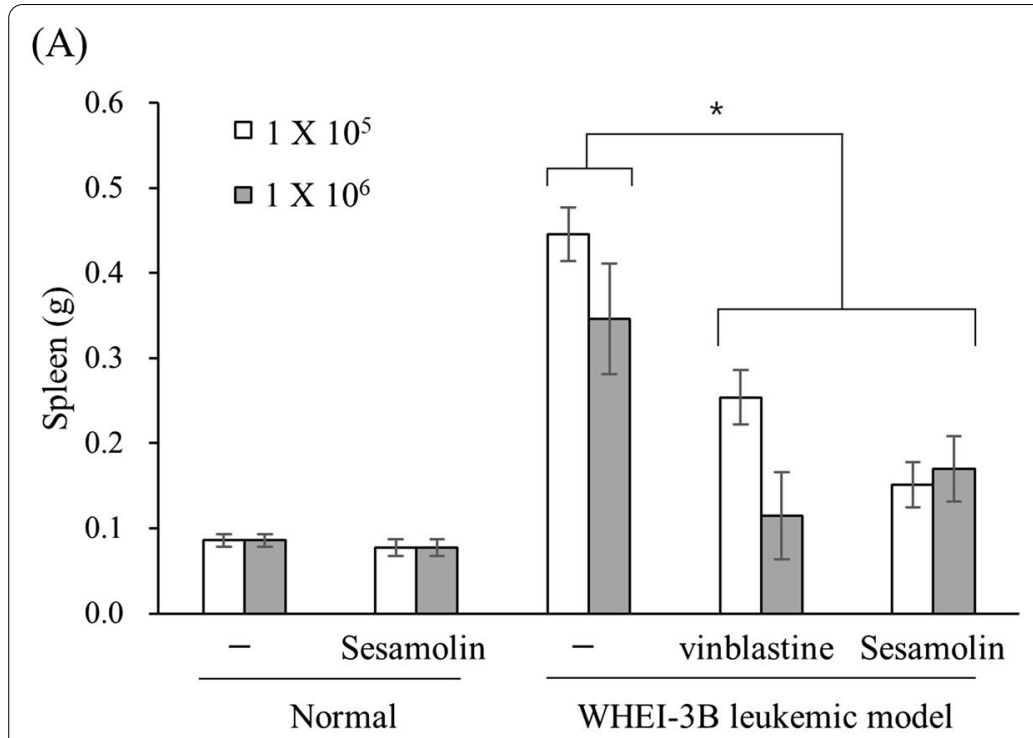

(C)

(B)
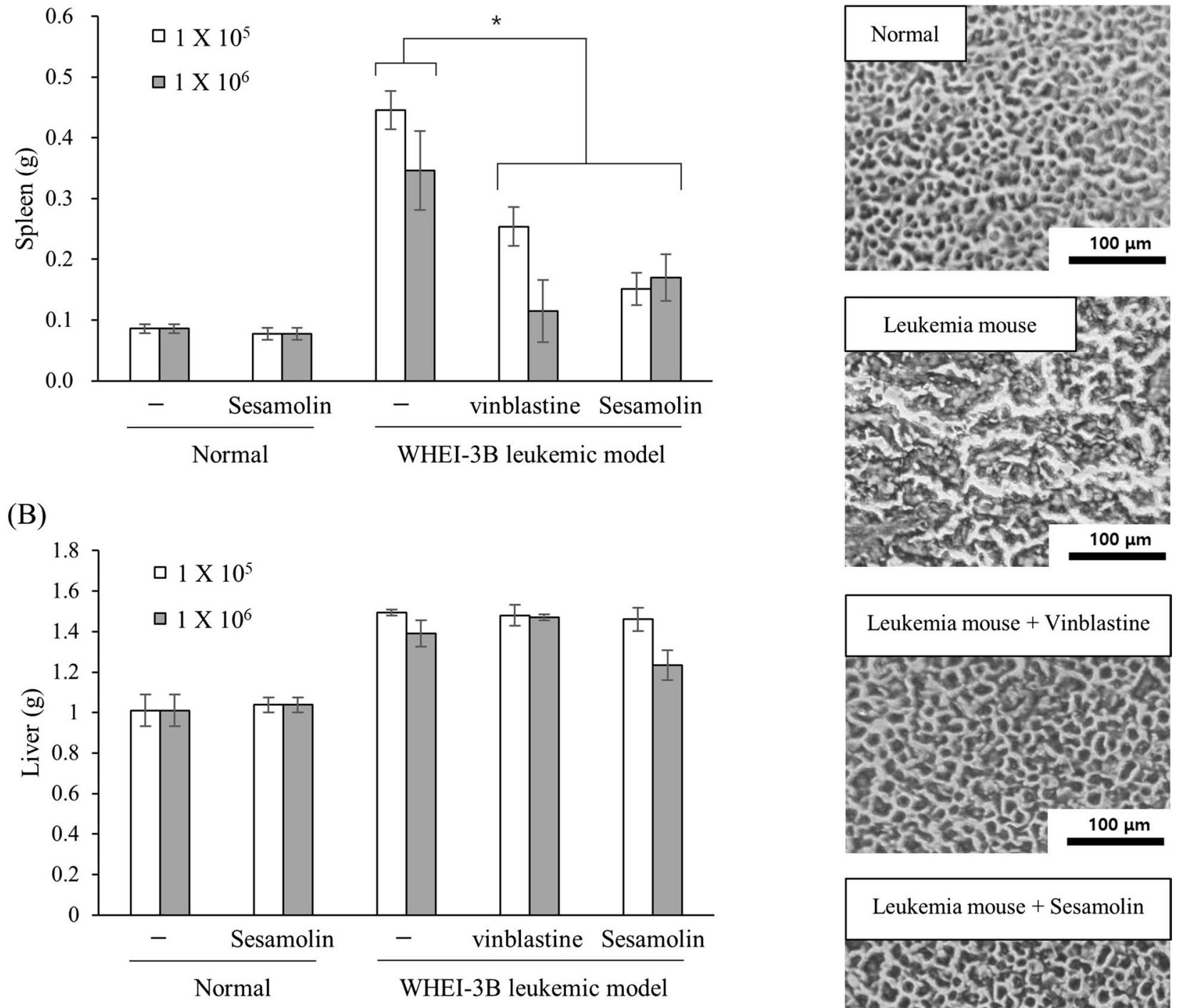

Leukemia mouse + Sesamolin

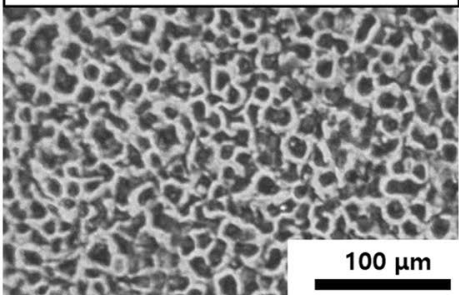

Fig. 4 Effects of sesamolin on internal organs. A, B Spleen and liver weights. Mice were injected with $1 \times 10^{5}$ or $1 \times 10^{6}$ WEHI-3B cells and administered with vinblastine, sesamolin, or neither for 3 weeks (3 doses per week). Spleen (A) and liver (B) weights. Data are representative of experiments repeated at least three times with triplicate samples and are shown as mean $\pm S D$. ${ }^{*} P<0.05$ vs. leukemic mice (one-way ANOVA with Dunnett's post-hoc tests). C Histopathological findings of fixed, embedded, and sectioned spleen tissues stained with H\&E and evaluated by microscopy at $400 \times$ magnification. Data are representative of experiments repeated at least three times

which their cytotoxic activity levels against Yac-1 and WEHI-3B cells were measured. It is shown in Fig. 8C that NK cells were more cytotoxic against WEHI-3B cells than against Yac-1 cells. Moreover, there was nearly a threefold increase in the cytotoxic activity of
NK cells against WEHI-3B, which was induced by sesamolin. These findings showed that sesamolin exerts anti-leukemic effects via the cytotoxic actions of NK cells in vivo and in vitro. 

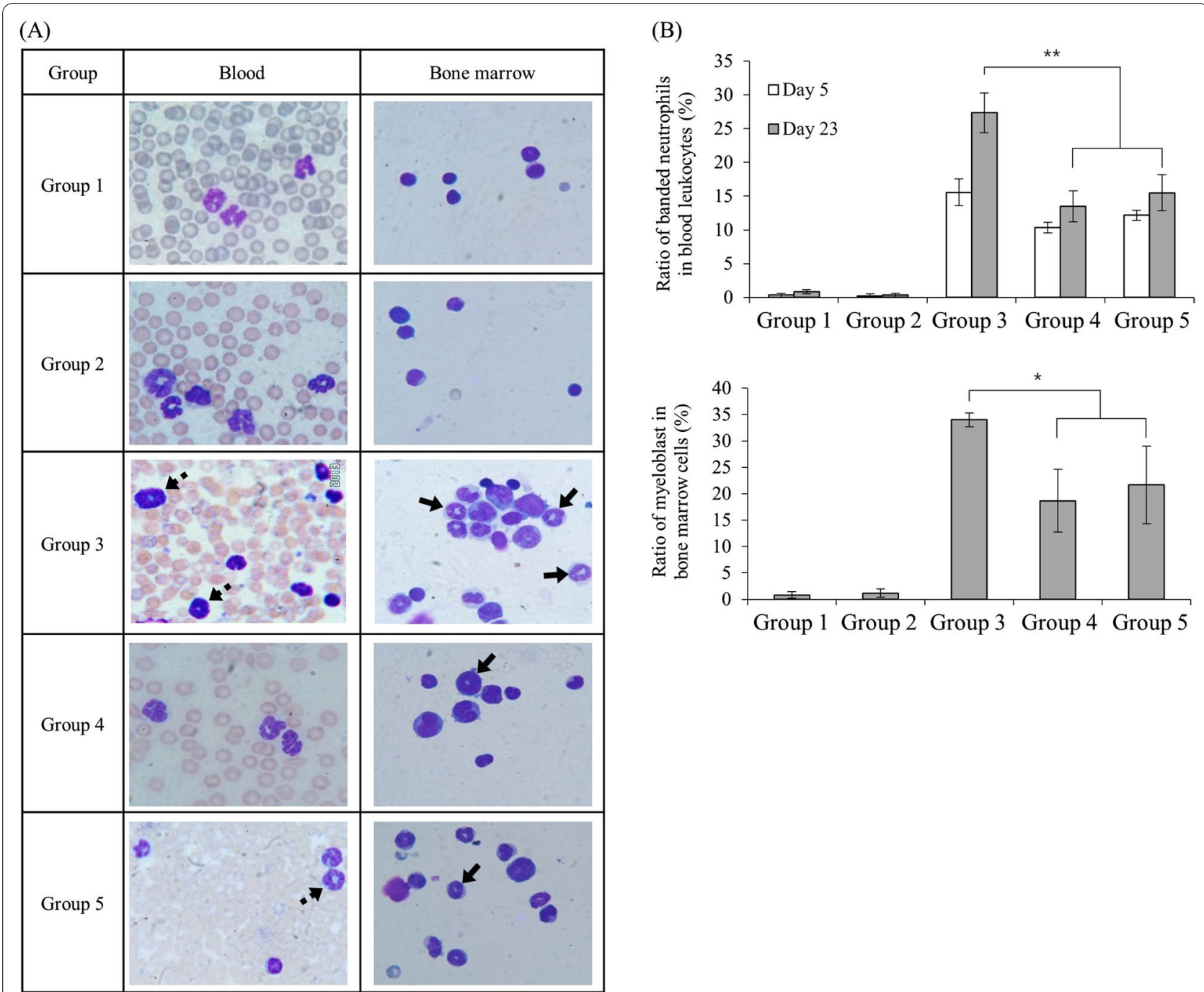

Fig. 5 Anti-leukemic effects of sesamolin at different time points. Normal and leukemic mice were classified according to the criteria listed in Table 2, and administered with sesamolin as enumerated in Table 3. A Mouse blood and bone marrow cells visualized with Wright Giemsa staining. Arrows $(\rightarrow$ indicate banded neutrophils and arrows $(\rightarrow$ ) indicate myeloblasts. Data are representative of experiments repeated at least three times. B Ratios (\%) of banded neutrophils in blood leukocytes and myeloblasts in bone marrow cells. Data are representative of experiments repeated at least three times with triplicate samples and are shown as mean $\pm S D$. ${ }^{*} \mathrm{P}<0.01,{ }^{*} \mathrm{P}<0.05 \mathrm{vs}$. group 3 (one-way ANOVA with Dunnett's post-hoc tests). Group 1, normal BALB/c mice; group 2, Normal BALB/c mouse administered with $10 \mathrm{mg} / \mathrm{kg}$ sesamolin (in CMC) from day 5; group 3, WEHI-3B-injected BALB/c mice; group 4, WEHI-3B-injected BALB/c mouse and administered with $10 \mathrm{mg} / \mathrm{kg}$ sesamolin in CMC from day 0; group 5, WEHI-3B-injected BALB/c mouse administered with $10 \mathrm{mg} / \mathrm{kg}$ sesamolin in CMC from day 5

\section{Discussion}

We previously showed that sesamolin exerts anticancer (anti-leukemia) activity in vitro [9]. Upregulated NKG2D ligand expression enhanced the sensitivity of sesamolin-treated Raji cells (leukemia cell line) to NK cell lysis via activation of the ERK signaling pathway. Moreover, sesamolin directly impacts NK cells [10]. The Raji cell-killing activities of NK cells increased depending on the sesamolin concentration. Sesamolin enhanced CD107a (degranulation marker) expression and interferon- $\gamma$ (immune-activation cytokine) production in NK cells. Sesamolin also produced an ideal environment that facilitated NK cells to kill leukemia cells [14]. The same study showed that sesamolin activates NK cells by regulating the differentiation and activation of dendritic cells (DCs) and that it could serve as a potential anticancer therapeutic agent in vitro. Therefore, we analyzed the anticancer activity of sesamolin in mouse models of leukemia induced by WEHI-3B cells in vivo. 


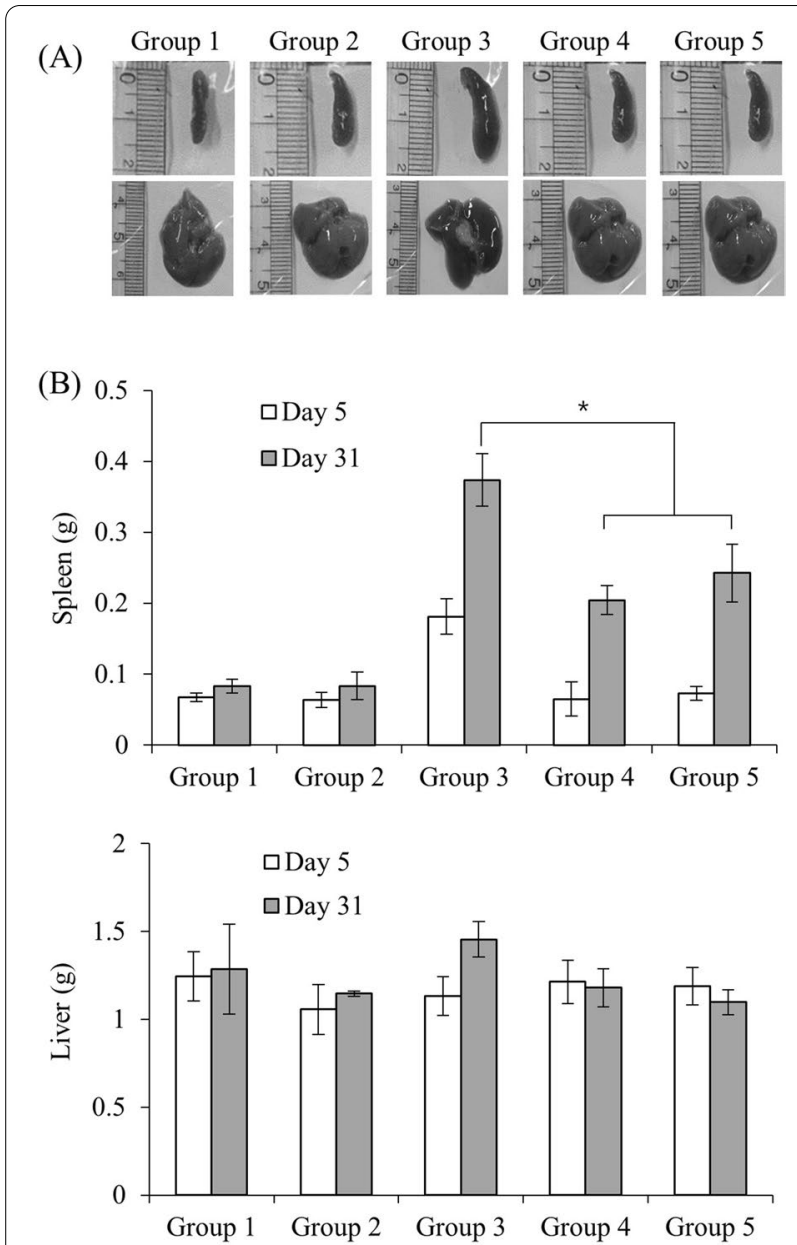

Fig. 6 Effects of sesamolin on liver and spleen weight. Effects of sesamolin on liver and spleen weight were assessed under the same experimental conditions as those illustrated in Fig. 5. A Photographs of spleen and liver. B Spleen and liver weights. Data are representative of experiments repeated at least three times with triplicate samples and are shown as mean $\pm S D$. ${ }^{*}<0.05$ vs. group 3 (one-way ANOVA with Dunnett's post-hoc tests). Group 1, normal BALB/c mice; group 2, normal BALB/c mice administered with $10 \mathrm{mg} / \mathrm{kg}$ sesamolin in CMC from day 5; group 3, WEHI-3B-injected BALB/C mice; group 4: WEHI-3B-injected BALB/C mice administered with $10 \mathrm{mg} / \mathrm{kg}$ sesamolin in CMC from day 0; group 5, WEHI-3B-injected BALB/C mice administered with $10 \mathrm{mg} / \mathrm{kg}$ sesamolin in CMC from day 5

We developed a model that demonstrated all the distinguishing features of myeloid leukemia by injecting BALB/c mice (i.p.) with WEHI-3B monomyelocytic leukemia cells that were originally derived from BALB/c mice [15]. To diversify the experimental results, two concentrations of WEHI-3B cells $\left(1 \times 10^{5}\right.$ and $1 \times 10^{6}$ cells/mouse) were injected in the first and second trials, respectively. The leukemic model mice exhibited the major characteristics of human myelomonocytic leukemia, such as the elevated levels of monocytes and granulocytes with immature morphology in peripheral blood, clearly enlarged spleen, and large numbers of immature cells in the bone marrow. [15]. Therefore, the WEHI-3B leukemia model is an established tool for screening novel anti-leukemia candidates [16]. The WEHI-3B cells aggressively invaded the soft tissue and blood-forming organs, such as the spleen and liver in our model. Organs that do not form blood and hardtissue organs such as the kidneys and heart did not exhibit altered characteristics in our model [16]. Our animal model was consistent with typical acute myeloid leukemia (AML), which is characterized by rapidly proliferating abnormal leukocytes, and showed splenomegaly along with increased numbers of immature granulocytes (banded neutrophil) and myeloblasts.

Retinoids are vitamin A metabolites that are representative natural anti-leukemia agents. Although they do not affect body weight, retinoids generate potent effects on the control of cellular differentiation and proliferation, and they often serve as positive controls for treating hemopoietic malignancies in leukemic animals [17]. Representative clinical signs of leukemia, such as splenomegaly and hepatomegaly in WEHI-3B leukemic mice, are mitigated by all-trans-retinoids [18]. Numerous anticancer compounds derived from natural products have been investigated in addition to retinoic acid.

The present study showed comparable therapeutic effects between sesamolin $(10 \mathrm{mg} / \mathrm{kg})$ and the positive control, vinblastine $(2.5 \mu \mathrm{g} / \mathrm{kg})$, in terms of reducing numbers of peripheral immature granulocytes and bone marrow myeloblasts, and decreasing the large and irregular spleen tissues in leukemic model mice. According to previously studies, sesamolin and vinblastine exhibited anti-leukemic effects at different concentrations [19, 20]. Therefore, in this study, sesamolin and vinblastine were administered at different doses based on these references. The natural lignan sesamolin is a minor component $(\sim 0.14 \%$ of the sesame oil by mass) of sesame seeds [7]. Sesamin is also a representative lignan derived from sesame seeds that has been investigated comprehensively as an anticancer agent more than sesamolin. Sesamin and sesamolin demonstrate similar chemical structures, but exert different anticancer effects [19]. Sesamin directly suppresses the proliferation of human K562 and KBM-5 leukemic cells, and of human myeloma U266 cells [21]. These suppressive effects of sesamin were realized via the tumor necrosis factor-mediated induction of NF- $\mathrm{BB}$ expression, which is responsible for leukemic cell responses [22]. 


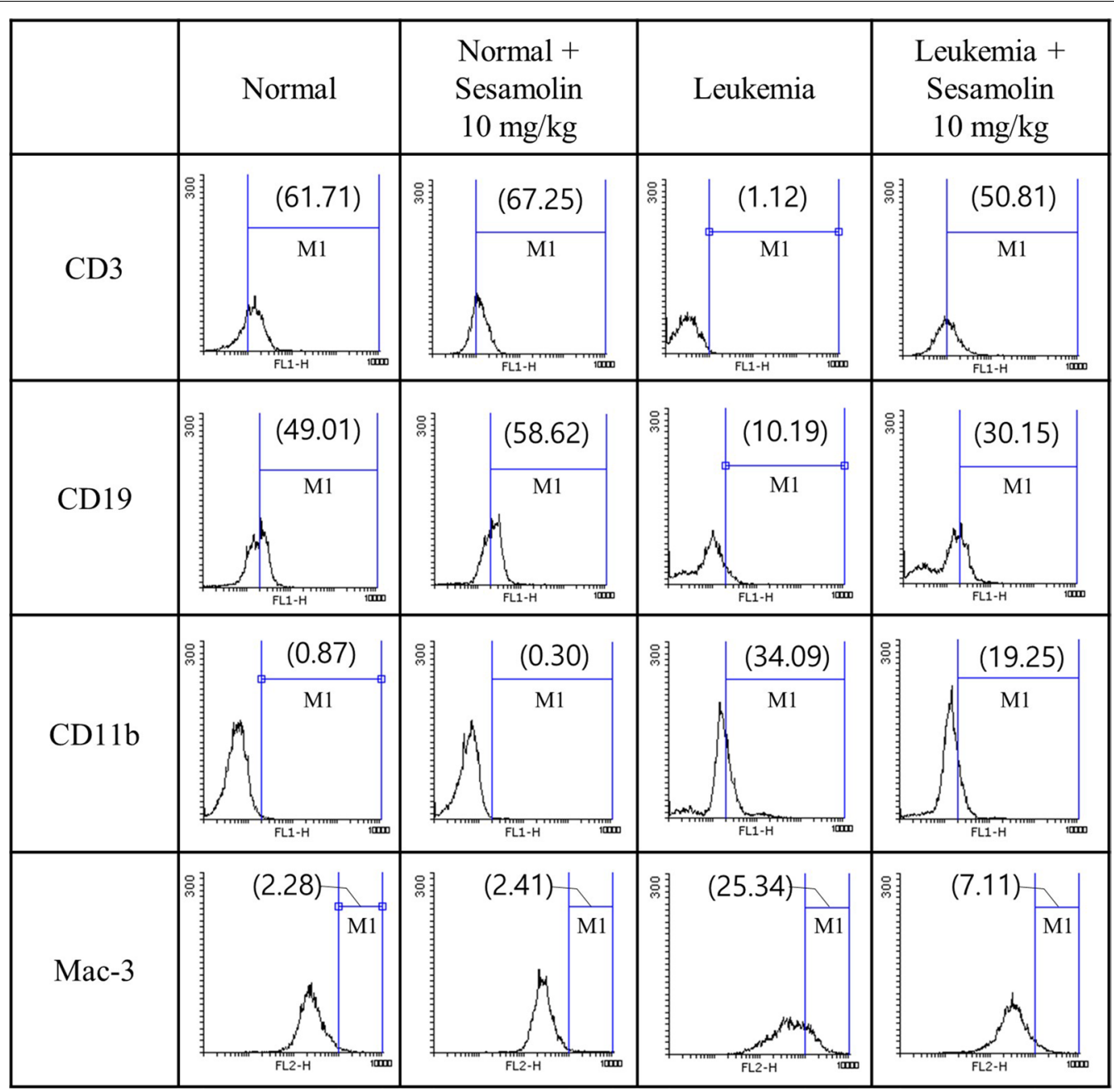

Fig. 7 Changes in leukocyte surface antigens in mice caused by induction of leukemia. Leukocytes separated from the blood of mice in groups 1 , 2, 3, and 5 (Table 2), were analyzed by FACS. Expression levels of CD3, CD19, CD1 1 b, and Mac-3 represented proportions of M1-gated cells among all leukocytes. Data are representative of experiments repeated at least three times

Sesamol is another lignan found in sesame oil that exerts anticancer effects in human leukemia cell lines. It induces growth inhibition and the apoptosis of Molt 4B human lymphoid leukemia cells [23] in a concentrationdependent manner. Tetrameric sesamol inhibits the proliferation of $\mathrm{K} 562$ cells in a concentration-dependent manner but is also cytotoxic against normal cells at active concentrations, thus rendering it unsuitable as a candidate anticancer agent [24]. Another lignan derived from sesame oil is sesaminol, which also exerts anti-leukemic effects on Molt 4B cells. Sesaminol ( $45 \mu \mathrm{M})$ inhibits Molt $4 \mathrm{~B}$ proliferation, and induces apoptosis of cancer cells $[19,25]$.

In summary, our results showed that sesamolin exerts anticancer effects against model mice with leukemia induced by WEHI-3B cells. The model mice exhibited more abundant banded neutrophils, myeloblasts, and monocytic leukemic cells than those observed in normal mice. Sesamolin reduced the number of leukemic cells in the model mice. The inhibitory effects of sesamolin and vinblastine (a positive control drug) on the proliferation of neoplastic cells were similar. Sesamolin reduced the weight of the leukemic mouse spleen to bring it within the normal range. Sesamolin also recovered the ratios of leukocytes altered by leukemia. Our results indicated that sesamolin possesses considerable potential as a novel anti-leukemia agent. However, we did not perform an experiment to determine the optimal concentration of sesamolin. In future studies, the anti-cancer activity of sesamolin will be tested at various concentrations to establish the optimal concentration for clinical application. 

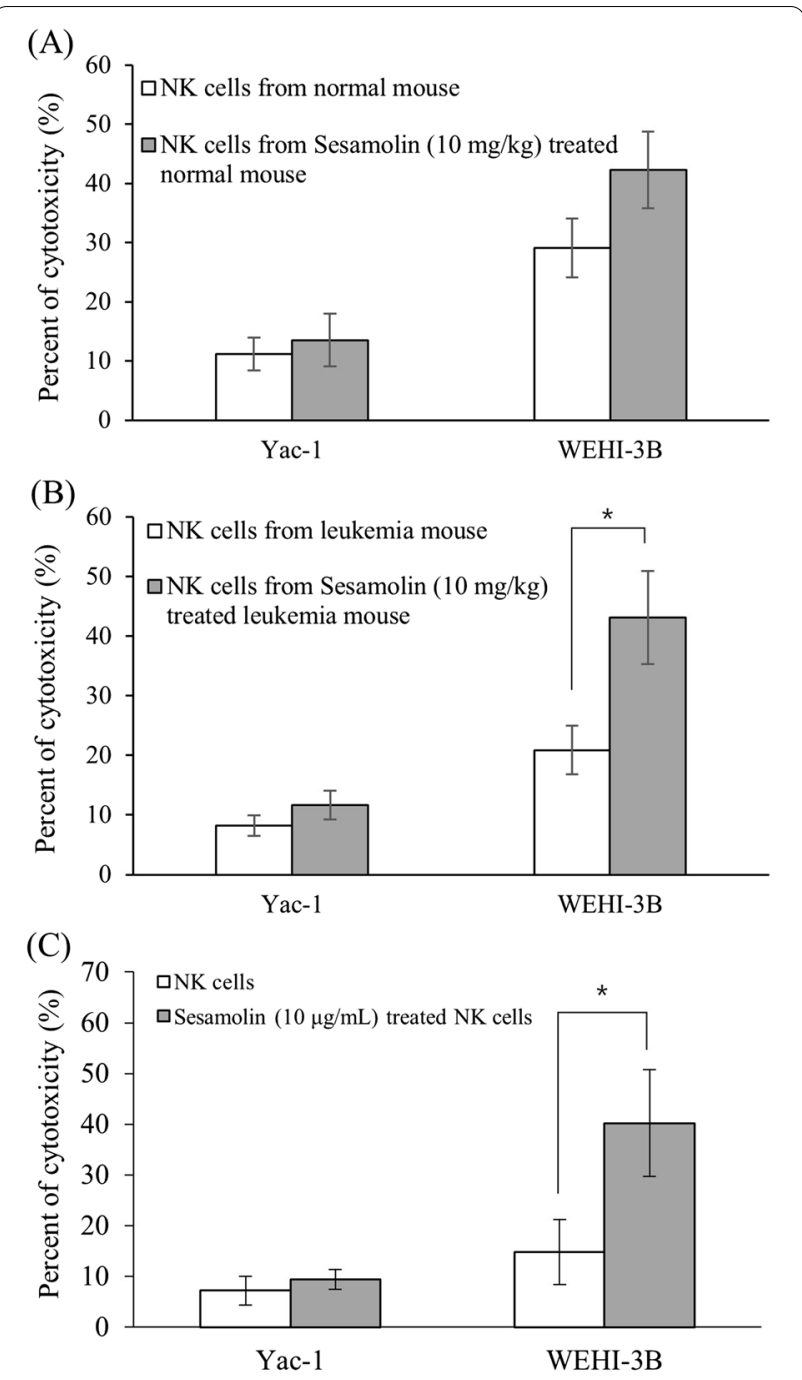

Fig. 8 Effect of sesamolin on the cytotoxic ability of naïve NK cells. NK cells derived from mice in groups 1,2,3, and 5 (Table 2) were used in LDH assays of Yac- 1 and WEHI-3B cells. Cytotoxic effects of NK cells derived from $\mathbf{A}$ normal and $\mathbf{B}$ leukemic mice administered with or without sesamolin for 3 weeks. C Effects of sesamolin on naïve NK cells in vitro. NK cells derived from normal mice were incubated with sesamolin $(10 \mu \mathrm{g} / \mathrm{mL})$ for $48 \mathrm{~h}$, and then cytotoxicity against Yac-1 and WEHI-3B was determined. Data are representative of experiments repeated at least three times with triplicate samples and are shown as mean $\pm S D$. ${ }^{*} P<0.05$ vs. NK cells from leukemic mice (one-way ANOVA with Dunnett's post-hoc tests)

\section{Acknowledgements}

This work was supported by the National Research Foundation of Korea (NRF) Grant funded by the Korea government (MSIT) (Grant No. 2019R1F1A1058970).

\section{Authors' contributions}

SN performed the majority of data analysis. JKL contributed to the writing of the manuscript and, planned and led this research. All authors read and approved the final manuscript.

\section{Declarations}

\section{Competing interests}

The authors declare that they have no competing interests.

\section{Author details}

'Department of Biology Education, College of Education, Chungbuk National University, Cheongju, Chungbuk 361-763, Republic of Korea. ${ }^{2} 204$, BEESEN BIO CO., Ltd, Bioventure Town, Yuseong Daero 1662, Dae Jeon, Republic of Korea.

Received: 9 December 2020 Accepted: 17 May 2021

Published online: 31 May 2021

\section{References}

1. Gutschner T, Diederichs S (2012) The hallmarks of cancer: a long noncoding RNA point of view. RNA Biol 9:703-719

2. Hanahan D, Weinberg RA (2000) The hallmarks of cancer. Cell 100:57-70

3. Greten FR, Grivennikov SI (2019) Inflammation and cancer: triggers, mechanisms, and consequences. Immunity 51:27-41

4. Kay J, Thadhani E, Samson L, Engelward B (2019) Inflammation-induced DNA damage, mutations and cancer. DNA Repair 83:102673

5. Clarke RT, Van Den Bruel A, Bankhead C, Mitchell CD, Phillips B, Thompson MJ (2016) Clinical presentation of childhood leukaemia: a systematic review and meta-analysis. Arch Dis Child 101:894-901

6. Mackey JBG, Coffelt SB, Carlin LM (2019) Neutrophil Maturity in Cancer Front Immunol 10:1912

7. Lim JS, Adachi Y, Takahashi Y, Ide T (2007) Comparative analysis of sesame lignans (sesamin and sesamolin) in affecting hepatic fatty acid metabolism in rats. Br J Nutr 97:85-95

8. Kumar CM, Singh SA (2015) Bioactive lignans from sesame (Sesamum indicum L.): evaluation of their antioxidant and antibacterial effects for food applications. J Food Sci Technol 52:2934-2941

9. Kim JH, Lee JK (2015) Sesamolin enhances NK cell lysis activity by increasing the expression of NKG2D ligands on Burkitt's lymphoma cells. Int Immunopharmacol 28:977-984

10. Lee SE, Lee JK (2018) Sesamolin affects both natural killer cells and cancer cells in order to create an optimal environment for cancer cell sensitization. Int Immunopharmacol 64:16-23

11. He Q, NaX (2001) The effects and mechanisms of a novel 2-aminosteroid on murine WEHI-3B leukemia cells in vitro and in vivo. Leuk Res 25:455-461

12. Alabsi AM, Ali R, Ideris A, Omar AR, Bejo MH, Yusoff K, Ali AM (2012) Anti-leukemic activity of Newcastle disease virus strains AF2240 and V4-UPM in murine myelomonocytic leukemia in vivo. Leuk Res 36:634-645

13. Chung JG, Yang JS, Huang LJ, Lee FY, Teng CM, Tsai SC, Lin KL, Wang SF, Kuo SC (2007) Proteomic approach to studying the cytotoxicity of YC-1 on U937 leukemia cells and antileukemia activity in orthotopic model of leukemia mice. Proteomics 7:3305-3317

14. Lee JK (2020) Sesamolin promotes cytolysis and migration activity of natural killer cells via dendritic cells. Arch Pharm Res 43:462-474

15. Tong ZZ, Fang ZM, Zhang Q, Zhan Y, Zhang Y, Jiang WF, Hou X, Li YL, Wang $T$ (2018) Plasmodium yoelii infection inhibits murine leukaemia WEHI-3 cell proliferation in vivo by promoting immune responses. Infect Dis Poverty 7:48-56

16. Tsou MF, Tien N, Lu CC, Chiang JH, Yang JS, Lin JP, Fan MJ, Lu JJ, Yeh SP, Chung JG (2013) Phenethyl isothiocyanate promotes immune responses in normal BALB/c mice, inhibits murine leukemia WEHI-3 cells, and stimulates immunomodulations in vivo. Environ Toxicol 28:127-136

17. Kiningham KK, Cardozo ZA, Cook C, Cole MP, Stewart JC, Tassone M, Coleman MC, Spitz DR (2008) All-trans-retinoic acid induces manganese superoxide dismutase in human neuroblastoma through NF-kappaB. Free Radic Biol Med 44:1610-1616

18. Ahmadi N, Rahman HS, Azmi Ibrahim T, Rosli R, KeongYeap S, Mohamed S (2017) Morinda citrifolia leaf extract ameliorated Leukemia in mice model. J Appl Biotechnol Bioeng 2(6):249-255 
19. Wu MS, Aquino LB B, Barbaza MY U, Hsieh CL, De Castro-Cruz K A, Yang LL, Tsai PW (2019) Anti-inflammatory and anticancer properties of bioactive compounds from Sesamum indicum L. A review. Molecules 4(24):4426-4454

20. Ali Salim LZ, Othman R, Abdulla MA, Al-Jashamy K, Ali HM, Hassandarvish P, Dehghan F, Ibrahim MY, Omer FA, Mohan S (2014) Thymoquinone inhibits murine leukemia WEHI-3 cells in vivo and in vitro. PLOS ONE 9(12):e115340

21. Harikumar KB, Sung B, Tharakan ST, Pandey MK, Joy B, Guha S, Krishnan S, Aggarwal BB (2010) Sesamin manifests chemopreventive effects through the suppression of NF-kappa B-regulated cell survival, proliferation, invasion, and angiogenic gene products. Mol Cancer Res 8:751-761

22. Siriwarin B, Weerapreeyakul N (2016) Sesamol induced apoptotic effect in lung adenocarcinoma cells through both intrinsic and extrinsic pathways. Chem Biol Interact 254:109-116
23. Miyahara Y, Hibasami H, Katsuzaki H, Imai K, Komiya T (2000) Sesamol induces apoptosis in human lymphoid leukemia molt $4 \mathrm{~B}$ cells. Food Sci Technol Res 6:201-203

24. Fujimoto A, Shingai Y, Oyama TB, Kawanai T, Hashimoto E, Koizumi K, Kimura K, Masuda T, Oyama Y (2010) Apoptosis-inducing action of two products from oxidation of sesamol, an antioxidative constituent of sesame oil: a possible cytotoxicity of oxidized antioxidant. Toxicol In Vitro 24:1720-1726

25. Namiki M (2007) Nutraceutical functions of sesame: a review. Crit Rev Food Sci Nutr 47:651-673

\section{Publisher's Note}

Springer Nature remains neutral with regard to jurisdictional claims in published maps and institutional affiliations.

\section{Submit your manuscript to a SpringerOpen ${ }^{\circ}$ journal and benefit from:}

- Convenient online submission

- Rigorous peer review

- Open access: articles freely available online

- High visibility within the field

- Retaining the copyright to your article

Submit your next manuscript at $\boldsymbol{\nabla}$ springeropen.com 